\title{
La profesión docente
}

\author{
MANUEL CASTILLO N.
}

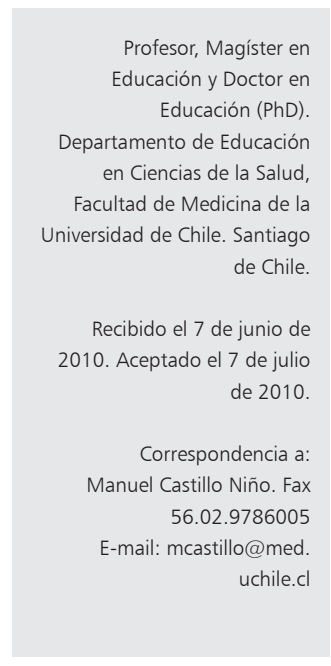

\section{The teaching profession}

This article focuses on understanding teaching activities, especially in the field of health science, as a set of tasks and actions that teachers undertake when transforming scientific knowledge into curriculum contents and actions. There are several ways to address this issue. Some authors identify specific roles and qualities of teachers structured as variables and dimensions. Others use the term competences, applied as skills, aptitudes or abilities to do something very well or duly intervene in a certain matter. The term competence, although not yet clearly defined, is used in this work because it is widely used in Health Sciences education. Some definitions are reviewed. Teacher's competences in the following areas recognized: those related to specific professional skills, in contents organization, in learning skills, in new information and communication technologies, in evaluation and in social and ethical areas of educational practices.

(Rev Med Chile 2010; 138: 902-907).

Key words: Faculty, medical; Models, educational; Teaching.
U no de los aspectos claves en el tema de la profesión docente consiste en dilucidar si existe o no una base de conocimientos de esta profesión que pueda considerarse "científica". En este ámbito existen dos grupos de conocimientos que pueden ser reconocidos. Por una parte los contenidos, esto es la disciplina que se enseñará, $y$, por otra, las capacidades relacionadas con los procedimientos para seleccionar, enseñar y evaluar esos contenidos. Esta combinación es compleja pues, por un lado, los contenidos disciplinarios no son meramente teóricos sino que su aprendizaje implica acciones prácticas y, por otra parte, la complejidad es mayor si consideramos que no cualquier procedimiento es adecuado para enseñar los distintos tipos de contenidos o disciplinas. Más bien, cada disciplina supone una didáctica y formas específicas de representación de sus contenidos y del trabajo con ellos.

La discusión acerca de si la docencia de los académicos que no son profesores puede ser considerada una profesión o no, es motivo de este artículo. De partida, la profesión docente reúne el más profundo sentido ético del concepto, que es desempeñarse o consagrarse a una causa de una gran trascendencia social y humana, donde la acción entre docente y estudiante va más allá del propio interés personal y da la oportunidad de entregarse seriamente en una causa educacional, que trasciende a quien la desempeña.

Este artículo da cuenta de algunas competencias o características del desempeño docente que hoy se reconocen como particulares y dan identidad a este ámbito de acción. Para esto es preciso iniciar conceptualizando el concepto de competencias docentes

\section{Las competencias}

Las competencias constituyen en la actualidad, una conceptualización y un modo de operar en la planificación y gestión de recursos humanos en la educación, orientada a facilitar una mejor articulación entre gestión, trabajo y educación ${ }^{2}$. Están relacionadas con una combinación integrada de conocimientos, habilidades y actitudes conducentes a un desempeño adecuado y oportuno en 
diversos contextos ${ }^{3}$. La flexibilidad y capacidad de adaptación resultan claves para el nuevo tipo de logro, que busca el trabajo y la educación como desarrollo general para que las personas hagan algo con lo que saben.

El término competencia puede ser definido de manera general como un saber hacer sobre algo, con determinadas actitudes y en determinados contextos ${ }^{4}$, es decir, como una expresión de lo que una persona puede hacer bien como resultado de la integración de sus conocimientos, habilidades, actitudes y cualidades personales 5 .

El término alude, en primer lugar, al carácter eminentemente práctico. Para saber si alguien es competente es indispensable observarlo actuando; es decir, no se es competente cuando sólo se sabe cómo se debe hacer, sino cuando se hace efectivamente y de una manera adecuada. En segundo lugar, la definición hace referencia a algo que se sabe hacer, que es el contenido de la competencia. En último lugar, para poder afirmar de alguien que es competente, no basta saber que hace ese algo, sino que importa mucho la manera o la actitud con que actúa ${ }^{6}$.

En este artículo se reconocen seis grupos de competencias y se recurre a diversos autores para aproximarse conceptualmente a cada área, estructurando una secuencia que a juicio del autor colabora a la comprensión del todo.

\section{Las competencias en la disciplina}

Indudablemente la capacidad de tratar y analizar problemas y fenómenos de una manera significativa requiere del dominio de un conjunto de conocimientos y habilidades de un área específica, el primer requerimiento intelectual de un profesional es el nivel en el que desarrolla su actividad. Pero "no solamente se refiere a la mera acumulación de estudios, sino acerca de las bases de ese contenido, su estructura, su significación y su dimensión social e histórica" [p. 220]1․

A pesar que cada vez se pone más énfasis en el cómo aprender y en el aprender a aprender, el qué aprender sigue estando en la base de la acción docente y no está en discusión que una competencia básica del profesional docente es un sólido conocimiento, un gran desarrollo de habilidades en su especialidad junto con una actitud de compromiso y entrega hacia ella.

Como en todas las profesiones, en las de la salud, la concepción de saber válido está en relación con una visión del conocimiento. Generalmente se asume que la verdad transmitida es objetiva, por tanto es lógico que el docente, que posee mejor nivel de conocimiento y de "verdad" que los alumnos ${ }^{7}$, tendrá prestigio por su experticia y reconocimiento como especialista, más que en sus méritos como docente ${ }^{8}$.

\section{Las competencias en la organización de los contenidos}

Diseñar la práctica pedagógica no sólo exige ordenar sus componentes para ser aprendidos por los alumnos, sino prever las condiciones de la enseñanza en el contexto educacional o fuera de él. La función más inmediata que deben desarrollar los docentes es la de diseñar o prever la práctica de la enseñanza [pag. 340]'

Diseñar la práctica es algo más amplio que atender a los objetivos y contenidos del currículo porque supone preparar las condiciones de su desarrollo a la vez que, también, se atiende a éste ${ }^{10}$, y ello supone establecer un puente para plasmar las ideas en realidad, un eslabón que conecta las intenciones y la acción. De acuerdo con las ideas que orientan ese puente, el proceso de enseñanza aprendizaje puede variar considerablemente ${ }^{9}$.

El docente se plantea la mejor manera de organizar los contenidos, pues tiene que ver, de alguna forma, con la reconstrucción de los conocimientos en los alumnos y, dada la amplitud del actual capital cultural, resulta difícil pensar en la comunicación cultural entre generaciones sin elaboraciones cuidadosas de la transmisión de esos contenidos ${ }^{11}$. Situándonos dentro de una disciplina, en un área o en la opción de relacionar áreas y disciplinas diversas, la forma de presentar organizados y agrupados los contenidos tiene enorme importancia, por cuanto las decisiones que se tomen condicionan, también, las construcciones cognitivas que puedan establecer los alumnos en su aprendizaje ${ }^{12}$.

De esta manera, una competencia docente importante tiene relación con la capacidad para organizar los contenidos. Una larga tradición pedagógica ha resaltado la importancia de ordenar los programas en torno a unidades didácticas, fórmula que supone distribuir el currículo en unidades con sentido en sí mismas, que pueden añadirse a otras para cubrir un área o un objetivo amplio o a largo plazo. Un problema no fácil de resolver es que la estructura del conocimiento 
supone establecer relaciones de dependencia entre las partes integrantes, dispuestas de tal manera que indiquen cuando el orden de la secuencia puede ser optativo o aleatorio y cuando ha de hacerse cuidadosamente para lograr una reconstrucción óptima ${ }^{13}$.

\section{Las competencias relacionadas con la variedad del aprendizaje}

Para planear la actividad docente es preciso considerar las habilidades de los estudiantes tomando en consideración su variedad de estilos de aprendizaje, o sea los rasgos cognitivos, afectivos y fisiológicos, que sirven como indicadores relativamente estables, de cómo los estudiantes perciben, interaccionan y responden a sus ambientes de aprendizaje $^{14}$.

$\mathrm{Al}$ respecto, se plantea ${ }^{8,14}$ que el aprendizaje es tarea del profesor en la medida que éste enseña a aprender, y que uno de sus roles reconocido es convertirse en un facilitador del aprendizaje. Este planteamiento deja claro que no basta que un docente sea experto en los contenidos, sino también debe serlo en la facilitación del aprendizaje. Si bien éste distribuye su tiempo en la enseñanza de contenidos y la enseñanza de estrategias de aprendizaje, esto último es una tarea relevante del docente por una doble razón: las estrategias que los estudiantes aplican a su aprendizaje influyen efectivamente en la calidad de éste, y tales estrategias son posibles de aprender y mejorables a través de la acción del docente.

Conocer el estilo de aprendizaje propio y el de los estudiantes, le permite al docente adaptar sus maneras de hacer como formadores, a las formas en que cada estudiante aprende mejor, y esto redundará en beneficio del aprendizaje ${ }^{15}$.

\section{Las competencias en la incorporación de nuevas tecnologías de la comunicación y de la información}

La universidad del siglo XXI tiene una fuerte proyección mediática; esto es, una referencia a las redes de comunicación; y cómo éstas son una herramienta que replantea la relación entre los procesos educativos en sus actuales lugares físicos, y sus relaciones de tiempo, entre la acción de enseñanza y la acción de aprendizaje, y, también de su materialidad, transformando en "bits" gran parte del material empleado para los estudios ${ }^{16}$.

No pareciera caber ninguna duda frente a la afirmación de Poole "el profesional docente universitario no puede iniciar un nuevo curso sin incorporar las nuevas tecnologías en sus planteamientos académicos, lo cual supone haber asumido todo un conjunto de conceptos y habilidades tecnológicas en su saber hacer común"17. El docente tiene, ahora, que incorporar un conjunto de recursos metodológicos nuevos que den cuenta de la aplicación racional y pertinente de las nuevas tecnologías, en el desarrollo del quehacer educativo ${ }^{18}$.

También, las tecnologías de la comunicación y de la información han puesto la educación a distancia al alcance del docente, quien necesita tener una visión de las potencialidades y limitaciones de esta modalidad educativa; debe conocer sus características particulares y diversas formas y estrategias que puede adoptar. Asimismo, conocer distintas formas organizativas para gestionar programas y proyectos educativos con modalidad a distancia o semi presencial.

La nueva sociedad que se está formando tiene mucho que decir sobre el cambio de costumbres y formas de entender el papel de las nuevas tecnologías. En el campo específico de la educación médica existen innumerables descripciones de experiencias relacionadas ${ }^{10,19-22}$. El desarrollo de estas aplicaciones presupone la conjunción de dos aspectos inseparables: la pedagogía y la tecnología y así es que las nuevas competencias en el diseño de estrategias de aprendizaje para los estudiantes son un requerimiento docente ${ }^{23}$.

\section{Las competencias en evaluación}

Con la finalidad de distinguir las prácticas de evaluación educativa diferenciando entre las modalidades tradicionales e innovadoras, recordaré el principio cognitivo básico que es que el aprendizaje requiere conocimientos, pero otro principio igualmente importante es que el conocimiento no puede transmitirse directamente a los alumnos, pues conocimiento es lo que ellos mismos elaboran, revisan, interpretan, cuestionan, confrontan con otras informaciones, relacionan con otros conocimientos, aplican a nuevas situaciones, razonan y, en definitiva, aprenden. Por lo que el rol del docente ya no es "dictar clase" y examinar a los alumnos, sino propiciar el desarrollo de conocimientos creativos y enseñarles estrategias de autorregulación y control sobre su proceso de autoaprendizaje; es decir, que los alumnos aprendan a aprender y a autoevaluarse sobre la marcha ${ }^{24}$. 
Entonces, la función evaluativa del profesor convencional varía radicalmente al cambiar la perspectiva de la enseñanza. Así, la evaluación del rendimiento académico de los estudiantes se ha definido tradicionalmente como la congruencia entre la respuesta solicitada a los estudiantes y el objetivo de aprendizaje propuesto ${ }^{25}$. De esta manera cuando el docente convencional evalúa, sus calificaciones tienen una función social en la medida que compara a cada alumno con el rendimiento del grupo e inevitablemente lo clasifica, lo selecciona y le pronostica de alguna manera su orientación futura. En un enfoque diferente, para formar a pensadores competentes, el docente tiene que empezar por resolver qué va a evaluar y también identificar y describir no sólo los tipos de aprendizajes categorizados según el grado de complejidad y de profundidad cognitiva, sino el uso de los procedimientos y estrategias autorreguladoras pertinentes durante el desarrollo del proceso de aprendizaje, y el avance logrado en la habilidad para pensar el tema objeto de enseñanza e, incluso, evaluar las estrategias comunicativas durante las actividades realizadas con el grupo ${ }^{24}$.

La literatura sobre la toma de decisiones del docente, resalta el hecho que las evaluaciones no se elaboran según un modelo teórico altamente estructurado, sino que tienen mucho que ver con "las apremiantes exigencias institucionales y con las demandas que la situación ambiental de clase le reclaman en un momento determinado" [p. 377 $]^{1}$.

Desde esta mirada, el fracaso de los estudiantes como expresión de una evaluación negativa está directamente ligado al cómo se realiza todo este proceso y a los esquemas que intervienen en él. Incluso puede plantearse la hipótesis de si no serán los propios procedimientos de evaluación los responsables del fracaso de los estudiantes ${ }^{1}$.

Una dificultad en el estudio de estas competencias es que el docente realiza el acto de evaluar de maneras muy distintas. Acceder a la privacidad de estos procedimientos en cada docente es bastante difícil, por la sencilla razón que la recogida de información sobre el trabajo y la conducta de los alumnos, la transformación de esa información y la emisión del juicio correspondiente es uno de los mecanismos más decisivos en la configuración de todo un estilo pedagógico personal, con fuertes concomitancias con el tipo de comunicación que mantienen con el alumnado. Estos mecanismos muchas veces se conciben más como pertenecien- tes a la esfera de lo íntimo, personal y oculto que a la estricta faceta profesional, pública y discutible.

La conducta de evaluación por parte de los docentes es una pauta de comportamiento profesional más, en la que los docentes se socializan. Se trata de una evaluación realizada de acuerdo con las requerimientos de una institución y, en condiciones concretas, no puestas por el evaluador ni por el evaluado.

\section{Competencias en el área de ética, en la acción educativa}

Aquí el término "competencias" adquiere una connotación especial, pues al referirnos a las complejas capacidades del docente para comprender el rol ético y social de la educación estamos en una dimensión profunda de su acción.

Es necesario revalorizar los aspectos éticos y culturales de la educación ${ }^{26}$; no es posible dejar de reflexionar con relación al hecho que muchas universidades se han convertido esencialmente en instituciones postsecundarias dedicadas a su entorno local y, en el mejor de los casos, se contentan con enseñar contenidos aislados unos de otros, se esfuerzan por conocer mejor a los hombres en su particularidad diversa y contradictoria, olvidándose del devenir global del mundo, de la armonía del hombre con la naturaleza y de la necesaria armonía entre los hombres para lograr la paz y la justicia social en forma efectiva; tanto más que el objetivo de la universidad no debiera ser tan sólo la universalidad de los conocimientos sino, además y sobre todo, la unidad del espíritu ${ }^{27}$.

El ser humano es esencialmente personal y comunitario a la vez. Desde esta perspectiva, satisface su naturaleza cuando establece relaciones de sentido con sus congéneres en un marco comunicacional, puesto que pertenece a su esencia el sercon-otro, el ser-por-otro y el ser-para-otro ${ }^{28}$. De esta forma compartir, recibir y dar constituyen una exigencia ética que lo realiza ${ }^{29}$. La educación, por ende, actualiza estas condiciones humanas al implicar con ello valores fundamentales, que deben surgir de la bondad y sabiduría de los educadores y reciprocarse en los educandos. La educación es un proceso de desarrollo interactivo, continuo, crítico y creativo, al considerar las dimensiones humanas en una perspectiva holística. La Ética, en cuanto ciencia normativa, regula necesariamente la actividad educacional, convirtiendo a la educación en la dimensión perfeccionadora de todas las otras. 


\section{Competencias en el área social, en la acción educativa}

El marco referencial para un análisis acerca del rol social de la acción docente lo plantearé desde los conceptos de un grupo de analistas sociopolíticos asociados con la escuela de Frankfurt, a la que pertenecen una serie de pensadores prominentes, de la talla de Habermas ${ }^{30}$. Todos ellos interesados en crear una sociedad más justa, transformando a las personas para que estén en una posición de mayor control sobre sus vidas económicas, políticas, sociales y culturales. Argumentan que esos objetivos pueden alcanzarse únicamente mediante la emancipación, proceso a través del cual las personas se constituyen en sujetos capaces de transformar, por sus propios medios, sus circunstancias ${ }^{30}$. Recibe el nombre de "teoría crítica", dado que ven la salida hacia la emancipación, a través de la toma de conciencia crítica que "problematiza" las relaciones sociales, en especial aquellas que se sitúan en las prácticas del ejercicio del poder, incluidas en los espacios educativos $^{31}$.

En resumen, la docencia constituye para muchos profesionales de la salud una segunda ocupación y las competencias, que he reconocido en este artículo, conforman algunos de los saberes disciplinares propios que la distinguen de otras. En esta reflexión aparece la necesaria integración entre la profesión de origen y la profesión docente. Con relación a esto surgen algunas preguntas ¿Existe una especificidad en cada profesión en el ámbito del diseño curricular, las didácticas o la evaluación?, ¿Hasta dónde puede existir una profesión sin que sus integrantes, necesariamente, se ocupen de reproducirla a través de escuelas de formación en institutos o universidades?

Todo parece indicar que es inseparable la función docente de la profesión original. Esto nos lleva a reconocer la especificidad de esas prácticas $y$, por lo tanto, reconocer la validez de las demandas que surgen desde las propias profesiones, relacionadas con la formación de los docentes formadores.

Quisiéramos contribuir con esta reflexión a abrir espacios a una serie de nuevas deliberaciones en torno a un tema que, en el ámbito de la imperativa renovación educacional, resulta no sólo providencial abordarlo, sino que estratégico y con visión de futuro, enfrentarlo.

\section{Referencias}

1. Torres J. El currículum oculto. Ediciones Morata. 1998, Madrid.

2. Rothewell WJ. Modelos para la Mejora del Rendimiento Humano, Funciones, Competencias y Resultados. Asociación Americana para la Formación y el Desarrollo. Madrid, 1996.

3. González C, Sánchez L. El diseño curricular por competencias en la educación médica. 2003: La Habana.

4. Saravia MA. Evaluación del profesor universitario. Un enfoque desde la competencia profesional, en Universitat de Barcelona http://www.tdx.cesca.es/. 2004: Barcelona.

5. Andrew G. Instrumentación de la educación basada en competencias., en Competencia laboral y educación basada en normas de competencia. Limusa, Editor. 1996: México.

6. McGaghie W, Miller G, Sajid A, Telder T. Introducción a la preparación de planes de estudios basados en la Competencia. Cuadernos de Salud Pública. OMS, 1998(68).

7. Bedoya JI. Epistemología y pedagogía. Ensayo histórico crítico sobre el objeto y métodos pedagógicos. Ecoe Ediciones. 2000, Colombia.

8. Castillo M. Perfil Docente de los académicos de la Facultad de Medicina, en DECSA Facultad de Medicina. 2003, Universidad de Chile: Santiago. p. 378.

9. Sacristán JG. El currículum: Una reflexión sobre la práctica., Ed. Morata. 1998, Madrid.

10. Westwood JD. Medicine meets virtual reality 11: Next Med: health horizon. Studies in health technology and informatics; v. 94. 2003, Amsterdam; Washington, DCTokyo: IOS; Ohmsha. xiii, 422 p.

11. Gijselaers W, Wilkerson L. Bringing problem-based learning to higher education: theory and practice. New directions for teaching and learning. 68. 1996, San Francisco: Jossey-Bass. 108 p.

12. Coll C, Martín E, Mauri T, Miras M, Onrubia J, Solé I, et al. El constructivismo en el aula. Ed. Prometeo. 2002, Buenos Aires.

13. Ausubel D. Psicología Educativa: Un punto de vista cognoscitivo. Ed. Ed. 1983, México. Trillas.

14. Alonso CM, Gallego DJ. Los estilos de aprendizaje. Procedimientos de diagnóstico y mejora., Ed. Mensajero. 2002, Bilbao.

15. Murillo P. Teorías sobre el cambio del profesorado. Paulino Murillo. Ed. Mergablu. 1999, Sevilla. 272.

16. Negroponte N. Ser Digital, Ed. Atlántida. 1995, Buenos Aires. 232.

17. Poole B. Tecnología Educativa. Educar para la sociocultura de la comunicación y del conocimiento. Ed. M.G. Hill. 1999, USA. 
La profesión docente - M. Castillo N.

18. Sánchez J. Informática Educativa. Ed. E. Universitaria. 2000, Santiago Chile. 179.

19. Manning PR, DeBakey L. Medicine, preserving the passion in the 21st century. 2nd ed. 2004, New York: Springer. $470 \mathrm{p}$.

20. Rice RE, Katz JE. The Internet and health communication: experiences and expectations. 2001, Thousand Oaks, Calif.: Sage Publications. xvi, 459 p.

21. Shneiderman B. Leonardo's laptop: human needs and the new computing technologies. 2002, Cambridge, Mass: MIT Press. xi, 269 p.

22. Haskell G, Rycroft MJ. Space and the global village: teleservices for the 21st century: proceedings of international symposium 3-5 June 1998, Strasbourg, France. Space studies; v. 3. 1999, Dordrecht; Boston: Kluwer Academic. xiv, 263 p.

23. Vitale B. La integración de la Informática en el aula, ed. A. Visor. 1994, Madrid.

24. Flores R. Evaluación pedagógica y cognición, ed. M.G.
Hill. 2000, México.

25. Himmel E. La evaluación escolar, Ed. Universitaria. 1972, Santiago.

26. Giroux HA. Postmodernism, feminism, and cultural politics: redrawing educational boundaries. 1991, Albany: State University of New York Press. x, 308.

27. Díez R. La reforma educativa en Ecuador, en El perfil del profesor universitario en los albores del siglo XXI, F.M. Sánchez, Editor. 1999: Quito.

28. Varela F. El fenómeno de la vida. Ed. Dolmen. 2000, Santiago.

29. Huaquín V. Ética y Educación Integral. Ed. D.d.I.C.y. Tecnológicas. 2001, Santiago Chile.

30. Habermas J. Ciencia y Técnica como Ideología. ed. Tecnos. 1984, México.

31. Magendzo A. Pedagogía crítica y educación en derechos humanos. Revista de Pedagogía crítica. Paulo Freire. Año $2 \mathrm{~N}^{\circ} 2,2003$. 